\title{
Generalized Quasi-Orthogonal Polynomials Applied in Sliding Mode-based Minimum Variance Control of ABS
}

\author{
Staniša Perić, Dragan Antić, Darko Mitić, Saša Nikolić, \\ Marko Milojković
}

University of Niš, Faculty of Electronic Engineering, Department of Control Systems, Aleksandra Medvedeva 14, 18000 Niš, Republic of Serbia stanisa.peric@elfak.ni.ac.rs, dragan.antic@elfak.ni.ac.rs, darko.mitic@elfak.ni.ac.rs, sasa.s.nikolic@elfak.ni.ac.rs, marko.milojkovic@elfak.ni.ac.rs

\begin{abstract}
This paper deals with the design of a digital sliding mode based minimum variance control on the basis of a discrete-time representation of the ABS model derived from a new type of generalized quasi-orthogonal filter. In the proposed control, the minimum variance enables the design of digital sliding mode control only on the basis of $A B S$ outputs measurements, while sliding mode increases the ABS robustness under certain conditions. On the other hand, it is shown that orthogonal functions can be successfully used to obtain a model of a dynamical system with high accuracy. The proposed control scheme has been applied in the laboratory experimental setup and obtained experimental results show significant improvement in ABS performances.
\end{abstract}

Keywords: sliding mode control; minimum variance control; orthogonal polynomials; antilock braking system

\section{Introduction}

Nowadays, we are witnessing the tremendous growth of the automotive industry in the world. Unfortunately, an increasing number of traffic accidents occur due to improper vehicle speed, sudden braking, bad road conditions, etc. The anti-lock braking system (ABS) is just one of the modern electronic systems found in vehicles, which contributes to the reduction of these accidents. It prevents the loss of control over the vehicle during sudden braking by disabling the vehicle wheels blocking in different road conditions (ice, snow, water, sand, etc.).

It has been shown that the ABS control problem can be solved by using different control approaches, starting from classical PID, through fuzzy logic and sliding 
mode controllers up to advanced control techniques based on the use of artificial neural networks, machine learning, etc. Herein, an overview of the most important contributions in sliding mode control (SMC) of ABS is given since this paper only deals with this type of control. In [1], SMC based on the exponential reaching law for ABS is developed to maintain the optimal slip value. The authors developed a two-wheel vehicle model in [2] and proposed an SMC algorithm to regulate ABS. The objective of [3] is to modify an optimal SMC method for hydraulic ABS in order to achieve both robustness and optimal control performances. SMC using a grey system theory approach for the ABS control is considered in $[4,5]$. The wheel slip control of the traction control system using a moving sliding surface is presented in [6]. One more approach of the moving sliding surface implementation for vehicle slip ratio control is shown in [7]. In [8], SMC on the basis of a two-axle vehicle model is discussed. In that paper, the authors also introduced the integral switching surface to cope with the chattering phenomenon. The similar approach of using the integral sliding surface is given in [9], but this time for the hybrid electric brake system. The traditional approach of the SMC design is applied in the control of the magnetorheological brake system in [10]. One more application of the traditional SMC is proposed in [11]. The second order sliding mode using the super-twisting technique to manipulate the braking torque is introduced in [12]. The quasi-continuous control for an automobile ABS is proposed in [13]. Therein, two controllers are developed, one to realize slip control and others for pressure tracking control. In [14], the same authors gave a more detailed analysis of the previous approach and compared the obtained results with the traditional SMC approach. Unlike the previous papers, where the wheel slip is used as a controlled variable, the authors of [15] considered a different approach based on the sliding mode by using the slip velocity. The latter approach is simulated on a "Magic formula" tire model.

From the previous analysis, it can be concluded that the further improvement of ABS performances can be realized by new control algorithms. Therefore, in this paper, an attempt will be made to achieve the optimum slip value by applying the novel control law that results from the combination of the sliding mode based minimum variance (MV) control and ABS modelling with orthogonal functions. It that way, the maximal value for the road adhesion coefficient is provided leading to better vehicle steering characteristics.

In the past several years the authors of this paper developed the new types of orthogonal filters [16], almost orthogonal filters [17, 18], improved almost orthogonal filters [19], quasi-orthogonal filters [20], orthogonal filters with complex zeros and poles [21] and generalized quasi-orthogonal filters [22]. These filters have proven to be a very powerful mathematical tool for modeling [18] and control of dynamical systems [16, 23, 24], as well as, for the approximation of real signals generated by industrial systems $[19,21]$. These filters can be also used to analyze the sensitivity of models of complex dynamical systems [25]. On the other hand, the signals generated by the generalized quasi-orthogonal filters of $k$-th 
order can be successfully applied as the activation functions of neural networks $[22,24]$. Moreover, it has been shown in [26] that these functions can replace the functions inside the layer that imitates Sugeno style defuzzification in the traditional ANFIS network. In [27], it has been already proved that orthogonal models, obtained by almost orthogonal filters, can be very effective for the design of SMC in the continuous-time domain. Herein, a similar concept for the new type of quasi-orthogonal filters, specially designed for this purpose, will be implemented, but this time in the discrete-time domain. The advantage of the proposed generalized quasi-orthogonal filters of shifted Müntz-Legendre type comes from the fact that they have the general values for poles in a transfer function, which significantly expands the possibility of their applications in comparison to the other, previously developed filters. This filter is used for obtaining the model of a plant, which will be employed then as a reference model in the design of SMC on the basis of MV control. The similar concepts of obtaining several linear and nonlinear models with successful applications in various fields including control are presented in [28, 29, 30].

The main goal of the combination of the sliding mode and MV control techniques is to improve the individual good characteristics of two control methods and to suppress their main drawbacks. The MV control can provide the realization of the digital sliding mode control (DSMC) only on the basis of the sensed system outputs. On the other hand, DSMC has been introduced to improve the robustness of MV control under the influence of external disturbances and parameter perturbations. It has been shown in [31] that digital sliding mode based MV control with accuracy $O\left(T^{2}\right)$ can be obtained by introducing the relay component into the control law, previously filtered through a digital integrator. The presence of a digital integrator significantly reduces the undesirable chattering phenomenon [32] providing a relatively smooth control signal in that way.

The laboratory setup of ABS is used in this paper [33]. The experimental results show the effectiveness of the proposed type of filters and control method in the field of modeling and control of ABS, respectively. The chosen laboratory framework proves to be very suitable for testing of different control algorithms $[34,35,36]$. By implementing the same control law with the previously derived model of ABS [27] and with the model derived in this paper, the obtained experimental results favour the latter control algorithm. In other words, the obtained orthogonal model describes the considered plant in a more efficient way due to the introduced parameter of imperfections in the very definition of the filter. From the control point of view, both experiments confirm the effectiveness of the proposed robust control method. The vehicle stopping time is further shortened with the preserved steering control, leading to the increased safety of the passengers.

The paper is organized as follows. Section 2 describes a new type of quasiorthogonal filter. In Section 3, the basic mathematical background of the proposed digital sliding mode based MV control is presented. The orthogonal model of ABS 
and modified control law in the case of ABS, are given in Section 4. The sensed outputs are presented and discussed in Section 5. Section 6 gives the most important concluding remarks.

\section{Generalized Quasi-Orthogonal Filters of Müntz- Legendre Type}

In this section, the generalized quasi-orthogonal filters of shifted Müntz-Legendre type (GQOFMLT), which contain an imperfections measure $\delta$ in their definition, are derived. This parameter actually describes imperfections of all the elements the system consists of, imperfections in the model, impact of the noise on the system output etc. [17, 22].

Now, let us consider a transfer function, $W_{n}^{(k, \delta)}(s)$, in the form suitable for the practical design of the proposed $k$-th order filter:

$$
W_{n}^{(k, \delta)}(s)=K \frac{\prod_{i=1}^{n-k}\left(s-p_{i} \delta\right)}{\prod_{i=0}^{n}\left(s+p_{i}\right)}=K \frac{\left(s-p_{1} \delta\right)\left(s-p_{2} \delta\right) \cdots\left(s-p_{n-k} \delta\right)}{s\left(s+p_{1}\right)\left(s+p_{2}\right) \cdots\left(s+p_{n}\right)},
$$

where $p_{i}$ represent poles of the transfer function, and $K$ is a gain of filter. The value of the constant $\delta$ is determined by performing several experiments so that it reflects the rate of parameters modification due to the changes in working temperature, humidity, etc. $[19,22]$. The main idea, herein, is to use this free parameter for description of non-modelled dynamics of a plant, obtaining in that way more faithful model representation. On the other hand, the effectiveness of control method heavily depends on model accuracy.

Development of (1) in partial fractions results in:

$$
W_{n}^{(k, \delta)}(s)=K \sum_{i=0}^{n} \frac{A_{n, i}^{(k, \delta)}}{\left(s+p_{i}\right)},
$$

where the coefficients $A_{n, i}^{(k, \delta)}$ are calculated as:

$$
A_{n, i}^{(k, \delta)}=K \frac{\prod_{\substack{j=1 \\ j \neq 0 \\ j \neq i}}^{n-k}\left(s_{j}-p_{i} \delta\right)}{\prod_{\substack{j \\ j}}^{n}\left(s_{j}\right)} .
$$


By using the transformation mapping $f(s)=\bar{s}=-s$ [16], the poles $p_{i}$ are being mapped into the zeros located in the right semi plane. After applying the inverse Laplace transform to (2), the sequence of orthogonal rational functions in time domain is derived as:

$\varphi_{i}^{(k, \delta)}(t)=\sum_{i=0}^{n} A_{n, i}^{(k, \delta)} e^{-p_{i} t}$

By taking $e^{-t}$ as a member $x$ of the polynomial, the latter relation can be rewritten as:

$L_{n}^{(k, \delta)}(x)=\sum_{i=0}^{n} A_{n, i}^{(k, \delta)} x^{p_{i}}$

where $L_{n}^{(k, \delta)}(x)=L^{-1}\left\{\varphi_{i}^{(k, \delta)}(t)\right\}$ represent the $k$-th order generalized quasiorthogonal polynomials of the shifted Müntz-Legendre type.

Note that if the poles $p_{i}$ in (2) have integer values, i.e., $p_{i} \in Z, i=0,1, \ldots, n$, someone can get the generalized quasi-orthogonal $k$-th order polynomials of Legendre type $P_{n}^{(k, \mathcal{S})}(x)$ [22], defined with the following expression:

$P_{n}^{(k, \delta)}(x)=\sum_{i=0}^{n} A_{n, i}^{k, \delta} x^{i}$

where:

$$
A_{n, i}^{k, \delta}=(-1)^{n+i+k} \frac{\prod_{j=1}^{n-k}(i+j \delta)}{i !(n-i) !} .
$$

The main difference between these two filter types lies in the fact that poles in the transfer function which correspond to (7) are fixed and a priori known. On the other hand, any real constant values for the poles can be selected in (1) causing in that way greater possibility of applications for the proposed type of filters.

On the basis of (1), it is easy to obtain the structure of the first order GQOFMLT $(k=1)$ which is very suitable for practical realization $[18,19,20]$. The general structure is shown in Figure 1, where Heaviside function is used as an input signal. 


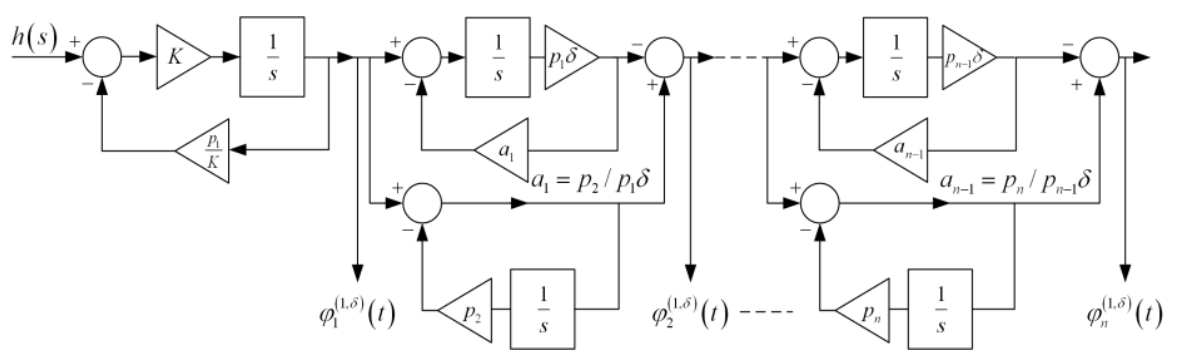

Figure 1

Schematic representation of the proposed filter for the first order $(k=1)$

It has been already shown that orthogonal signals $\varphi_{i}^{(1, \delta)}(t)$, generated using the orthogonal filters, are appropriate for the analysis and synthesis of different technical systems $[21,22,25]$. In this case, the parameters of filter $\left(K, p_{i}\right.$, and $\left.c_{i}\right)$ are adjusted to minimize the value of the mean squared error:

$J=\frac{1}{T} \int_{0}^{T}\left(y_{S}(t)-y_{M}(t)\right)^{2} d t$,

where $y_{S}(t)$ is a real system output and $y_{M}(t)$ represents an output of the orthogonal model described by the following expression:

$y_{M}(t) \approx \sum_{i=0}^{n} c_{i} \varphi_{i}^{(1, \delta)}(t)$.

On the basis of these plant models and their representation in the discrete-time domain, a digital sliding mode controller is designed in the next section. The model parameters are obtained by identification based on the measured ABS responses, using some of the optimization techniques. The procedure itself is briefly described in Section 4, while the entire modelling process, using genetic algorithms as an optimization method, is thoroughly described in $[18,19]$.

\section{Digital Sliding Mode-based Minimum Variance Control}

Let us consider a continuous-time SISO plant model in the following form:

$$
\begin{aligned}
\dot{\mathbf{x}}(t) & =\mathbf{A x}(t)+\mathbf{b} u(t)+\mathbf{d} f(t), \\
y(t) & =\mathbf{c} \mathbf{x}(t),
\end{aligned}
$$


where $\mathbf{x}(t)=\left[\begin{array}{llll}x_{1}(t) & x_{2}(t) \quad \ldots \quad x_{n}(t)\end{array}\right]^{T} \in R^{n}$ is a vector of state coordinates, $u(t) \in R$ represents a plant input, $f(t) \in R$ is an external disturbance, $y(t) \in R$ denotes a plant output, $n$ determines plant order, and matrix $\mathbf{A}$ and vectors, $\mathbf{b}$, $\mathbf{c}$ and $\mathbf{d}$ are with the following dimensions: $\mathbf{A}=\left[a_{i j}\right]_{n \times n}, \mathbf{b}=\left[b_{i}\right]_{n \times 1}, \mathbf{c}=\left[c_{j}\right]_{1 \times n}$, $\mathbf{d}=\left[d_{i}\right]_{n \times 1}$. This model can be directly derived from (9) taking into account (1)(4).

The discrete-time model of (10) is given by:

$$
\begin{aligned}
& \dot{\mathbf{x}}_{k+1}=\boldsymbol{\phi} \mathbf{x}_{k}+\gamma u_{k}+\mathbf{h}_{k}, \\
& y_{k}=\mathrm{c} \mathbf{x}_{k},
\end{aligned}
$$

whereas:

$\phi=e^{\mathbf{A} T}$,

$\boldsymbol{\gamma}=\int_{0}^{T} e^{\mathbf{A} \tau} \mathbf{b} d \tau$

$\mathbf{h}_{k}=\int_{0}^{T} e^{\mathbf{A} \tau} \mathbf{d} f((k+1) T-\tau) d \tau$.

To enhance further writing, the following notation $\bullet_{k}=\bullet(k T)$ is accepted, where $T$ denotes the sampling period. The external disturbance $f(t)$ is supposed to be a bounded function, i.e., there exists a constant $F<\infty$ such that $|f(t)|<F$. On the basis of (11), the plant model in the $z$-domain can be obtained as:

$$
y_{k}=\frac{z^{-1} B\left(z^{-1}\right)}{A\left(z^{-1}\right)} u_{k}+\frac{z^{-1} \mathbf{D}\left(z^{-1}\right)}{A\left(z^{-1}\right)} \mathbf{h}_{k},
$$

where $z^{-1}$ represents a delay operator, i.e., $z=e^{p T}$ ( $p$ is a complex variable), and:

$$
\begin{aligned}
& A\left(z^{-1}\right)=z^{-n} \operatorname{det}(z \mathbf{I}-\boldsymbol{\phi}), \\
& B\left(z^{-1}\right)=z^{-n+1} \mathbf{c}[\operatorname{adj}(z \mathbf{I}-\boldsymbol{\phi}) \boldsymbol{\gamma}], \\
& \mathbf{D}\left(z^{-1}\right)=z^{-n+1} \mathbf{c}[\operatorname{adj}(z \mathbf{I}-\boldsymbol{\phi})] .
\end{aligned}
$$

The main objective of the designed control is to ensure minimum variance of the variable:

$$
s_{k}=M\left(z^{-1}\right)\left(y_{k}-r_{k}\right),
$$


i.e., in an ideal case $s_{k}=0$. In addition, the polynomial $M\left(z^{-1}\right)$ is a Jury's polynomial, and $r_{k}$ is a reference input signal in the $k$-th time period. The plant output in the steady state can be defined with:

$$
y_{\infty}=r_{\infty}+\frac{s_{\infty}}{M(1)} .
$$

From the last equation it can be concluded that the accuracy of the system output will depend only on the accuracy of the variable $s_{k}$. Therefore, by keeping the smallest value of $s_{k}$, the smallest possible tracking error will be achieved.

To accomplish the above-mentioned control goal, the digital sliding mode based MV control is proposed in the following form [31]:

$$
u_{k}=-\frac{1}{E\left(z^{-1}\right) B\left(z^{-1}\right)}\left(F\left(z^{-1}\right) y_{k}-M\left(z^{-1}\right) r_{k+1}+\frac{\alpha T}{1-z^{-1}} \operatorname{sgn}\left(s_{k}\right)\right) \text {, }
$$

where $E\left(z^{-1}\right)$ and $F\left(z^{-1}\right)$ are the solutions of Diophantine equation:

$$
E\left(z^{-1}\right) A\left(z^{-1}\right)+z^{-1} F\left(z^{-1}\right)=M\left(z^{-1}\right),
$$

with assumption that $r_{k}$ is known in advance. The digital integrator (in the front of sgn $\left(s_{k}\right)$ ) in (19) should alleviate the chattering phenomenon [32, 37]. By substituting (19) in (13), and taking into account (17) and (20), the switching function dynamics can be obtained as:

$$
s_{k+1}=s_{k}-\alpha T \operatorname{sgn}\left(s_{k}\right)+E\left(z^{-1}\right) \mathbf{D}\left(z^{-1}\right)\left(\mathbf{h}_{k}-\mathbf{h}_{k-1}\right),
$$

where parameter $\alpha$ should provide stable quasi-sliding motion and it is chosen in accordance with the following theorem.

Theorem: Let us consider the system described by (13) and (19), where the switching function and its dynamic is given by (17) and (21), respectively. If the parameter $\alpha$ is chosen to satisfy:

$$
\alpha T>\max \left|E\left(z^{-1}\right) \mathbf{D}\left(z^{-1}\right)\left(\mathbf{h}_{k}-\mathbf{h}_{k-1}\right)\right|,
$$

then the control (19) forces a system phase trajectory to reach the quasi-sliding manifold $S$ determined by:

$$
S=\left\{s_{k}:\left|s_{k}\right|<\alpha T+\max \left|E\left(z^{-1}\right) \mathbf{D}\left(z^{-1}\right)\left(\mathbf{h}_{k}-\mathbf{h}_{k-1}\right)\right|\right\},
$$

in finite time and keeps it on it for every $k>K_{0}$, where $K_{0}=K_{0}\left(s_{0}\right)$ is a positive number.

The detailed proof of this Theorem could be found in [38]. 


\section{A Case Study: Anti-Lock Braking System}

As a case study, a laboratory ABS, presented in Figure 2 has been chosen, because it is suitable for the practical verification of the proposed modelling and control approaches due to its strong nonlinear nature [39, 40, 41].

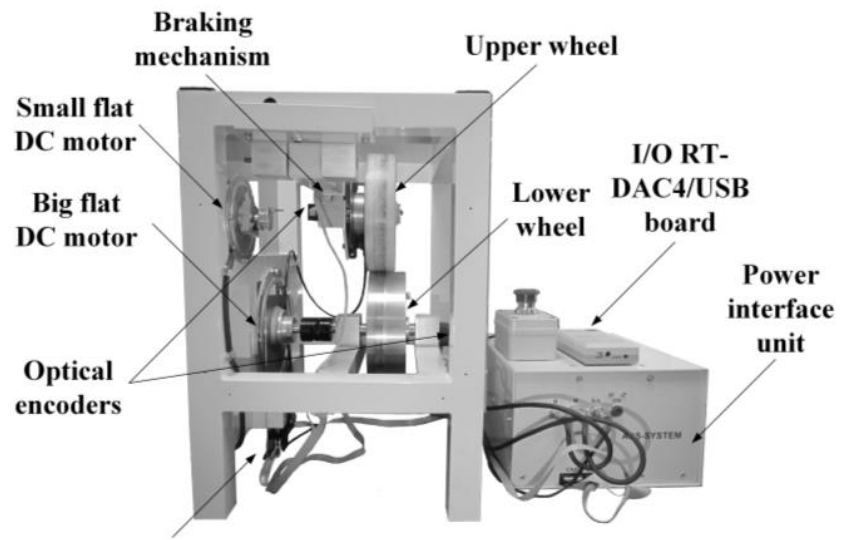

Brake lever

Figure 2

Laboratory test setup of ABS

The complete mathematical and physical description of this system can be found in our earlier papers [24, 42] and it is derived on the basis of graphical representation of ABS setup presented in Figure 3.

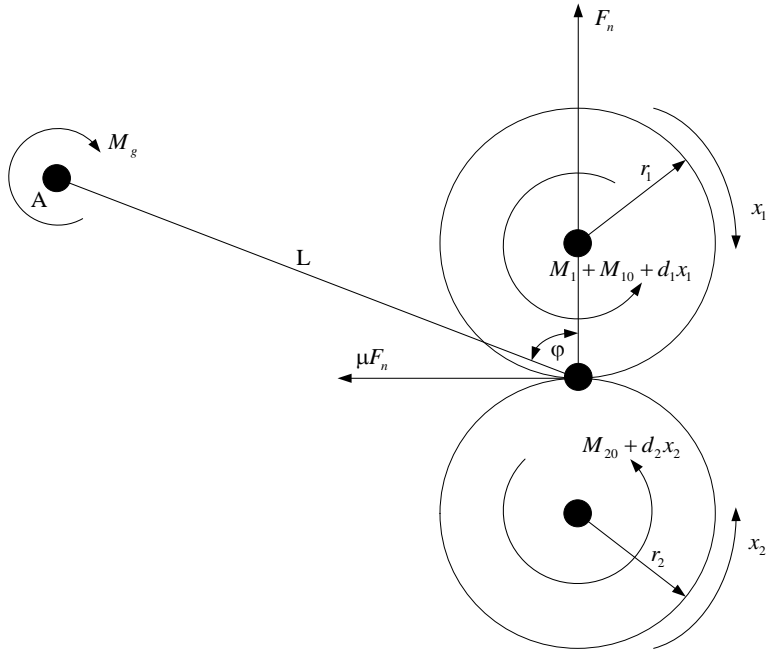

Figure 3

Schematic diagram of the experimental setup 
Based on this first-order nonlinear model, the DSMC has been designed in [42].

In [43], it has been shown that the dynamics of ABS can be represented well enough by the second-order transfer function with finite zero:

$W(s)=\frac{\bar{b}_{1} s+\bar{b}_{0}}{s^{2}+\bar{a}_{1} s+\bar{a}_{0}}$

where wheel slip and braking moment are used as system output and input, respectively. In order to control such minimum-phase plant, the starting model (27) is transformed into the controllable canonical form first and then divided into two subspaces [44]:

$\dot{x}_{1}=d_{11} x_{1}+d_{12} x_{2}+k u$,

$\dot{x}_{2}=d_{21} x_{1}+d_{22} x_{2}$,

$y=x_{1}$,

On the basis of the latter model, SMC is designed for the upper subsystem (28) of the first order in [27]. Notice that the control approaches presented so far use the first-order model of ABS for design purposes. It should be expected that the use of the second-order model of ABS in controller design would give better results.

In this paper, several real-time experiments have been performed and wheel slip for different values of braking moment has been recorded. After that, the parameters of the second-order model (27) have been identified by using the classical approach as $\bar{a}_{0}=0.7708, \bar{a}_{1}=74.3301, \bar{b}_{0}=0.0059$, and $\bar{b}_{1}=0.6840$. This model will be used further for the validation purposes of the proposed control approach. The same experimental results are used to obtain the suggested orthogonal model parameters. Based on the plant response, it has been concluded that the ABS model can be described by proposed GQOFMLT with two sections according to (1), (4), and (8). As mentioned earlier, for parameters adjustment of filter ( $K, p_{1}, p_{2}, c_{0}, c_{1}$, and $c_{2}$ ) the genetic algorithm [19,21,22] can be used. Herein, the genetic algorithm has a chromosome consisting of 6 parameters coded by real numbers (filter parameters) and fitness function (8). After the parameters adaptation procedure, $K=0.0065, \quad p_{1}=76.9230, \quad p_{2}=0.0103, \quad c_{0}=0.00000047$, $c_{1}=109.44429, c_{2}=0.0000120548, J_{\min }=2.312654 \cdot 10^{-8}$ are obtained. Simulation time is $20 \mathrm{~s}$. The value of parameter $\delta$ has been chosen to be 1.002837 . The parameter $c_{0}$ can be neglected because its value is very close to zero.

Now, the state-space model of ABS can be obtained in the form (10), where: 


$$
\begin{aligned}
& \mathbf{A}=\left[\begin{array}{cc}
0 & 1 \\
-p_{1} p_{2} & -\left(p_{1}+p_{2}\right)
\end{array}\right], \\
& \mathbf{b}=\left[\begin{array}{l}
0 \\
1
\end{array}\right], \\
& \mathbf{c}=\left[\begin{array}{ll}
c_{1}+c_{2} & \left.K\left(c_{1} p_{2}-c_{2} p_{1} \delta\right)\right], \\
\text { and } \mathbf{d}=[0] .
\end{array}\right.
\end{aligned}
$$

Using (12) the discrete-time ABS model can be derived in the form (11) with:

$$
\begin{aligned}
\phi & =\left[\begin{array}{cc}
-0.7 e^{-64 T}+1.1 e^{-4.2 T} & -0.01 e^{-64 T}+0.02 e^{-4.2 T} \\
-268.331\left(-0.01 e^{-64 T}+0.02 e^{-4.2 T}\right) & 1.07 e^{-64 T}-0.07 e^{-4.2 T}
\end{array}\right], \\
\gamma & =\left[\begin{array}{c}
0.003+0.0003 e^{-64 T}-0.004 e^{-4.2 T} \\
3.46 \times 10^{-18}-0.02 e^{-64 T}+0.02 e^{-4.2 T}
\end{array}\right],
\end{aligned}
$$

and, finally, the ABS model in the form of discrete-time transfer function is:

$$
\lambda_{k}=\frac{z^{-1} B\left(z^{-1}\right)}{A\left(z^{-1}\right)} M_{1 k}
$$

where the coefficients of the polynomials $A\left(z^{-1}\right)$ and $B\left(z^{-1}\right)$ are $a_{0}=1$, $a_{1}=-1.4861, a_{2}=0.5055, b_{0}=0.008$ and $b_{1}=-0.0015$ for $T=10 \mathrm{~ms}$.

In the case of ABS, the digital sliding mode based MV control (19) is given by:

$$
M_{1 k}=-\frac{1}{E\left(z^{-1}\right) B\left(z^{-1}\right)}\left(F\left(z^{-1}\right) \lambda_{k}-M\left(z^{-1}\right) \lambda_{k+1}^{r e f}+\frac{\alpha T}{1-z^{-1}} \operatorname{sgn}\left(s_{k}\right)\right),
$$

The proposed control algorithm should provide zero value of the switching function:

$$
s_{k}=M\left(z^{-1}\right)\left(\lambda_{k}-\lambda_{k}^{r e f}\right),
$$

with $M\left(z^{-1}\right)=m_{o}+m_{1} z^{-1}+m_{2} z^{-2}$ where $m_{0}=1, m_{1}=-1.0670, m_{2}=0.2846$. The coefficients of the polynomial $M\left(z^{-1}\right)$ are calculated by using $M\left(z^{-1}\right)=\left(z-\exp \left(-2 \pi f_{c} T\right)\right)^{2}$ where $f_{c}=10 \mathrm{~Hz}$ is a cut-off frequency. According to (20), the polynomials $E\left(z^{-1}\right)$ and $F\left(z^{-1}\right)$ are defined in this case as:

$$
E\left(z^{-1}\right)=e_{0}=\frac{m_{0}}{a_{0}}
$$


$F\left(z^{-1}\right)=f_{0}+f_{1} z^{-1}$

$f_{0}=m_{1}-e_{0} a_{1}$,

$f_{1}=m_{2}-e_{0} a_{2}$.

In order to compare the results obtained by using the proposed control approach based on the ABS orthogonal model, the same control law (32)-(35) has been implemented by using the discrete-time representation of (27) in the form of (31).

\section{Experimental Results}

To verify the effectiveness of the proposed type of filters and control algorithm designed on the basis of sliding mode and MV, two experiments have been done on ABS experimental setup [33]. First, one has been performed by using the digital sliding mode based MV control (32) designed on the basis of the discretetime representation (13) of ABS model (27) and its results are shown in Figure 4. The second experiment has been realized by using the proposed control law (32) developed by using the discrete-time representation (31) of ABS orthogonal model (29) described with GQOFMLT, and the obtained results are presented in Figure 5. In this way, by implementing the same control law and different referent models in their design, it can be concluded whose model better describes the ABS dynamics. In both cases, the run time is $2.7 \mathrm{~s}$, the sampling period $T=10 \mathrm{~ms}$, the reference wheel slip $\lambda_{k+1}^{r e f}=0.2$ [45], and the controller parameter $\alpha$ is chosen to be 1 .
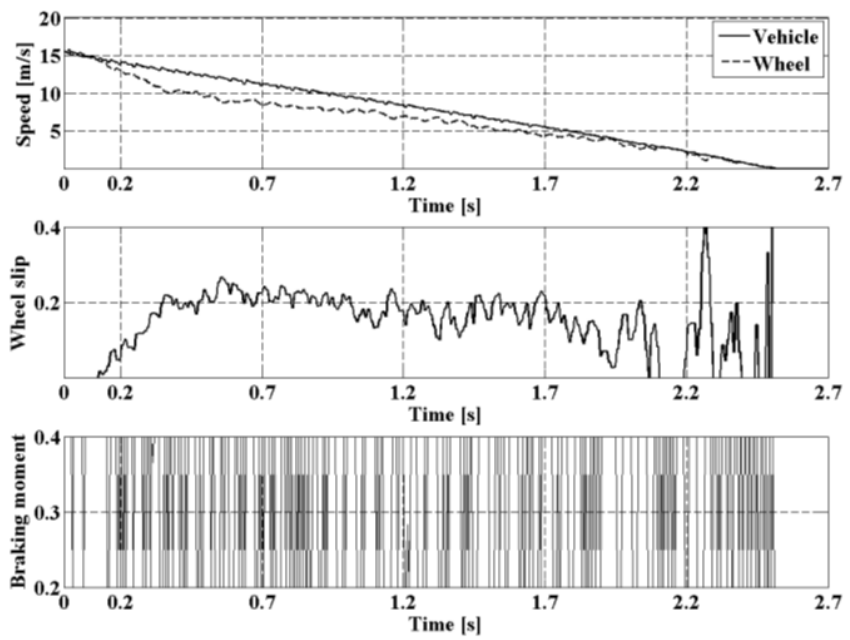

Figure 4

ABS responses with the control law (32) derived from the ABS model (27) 

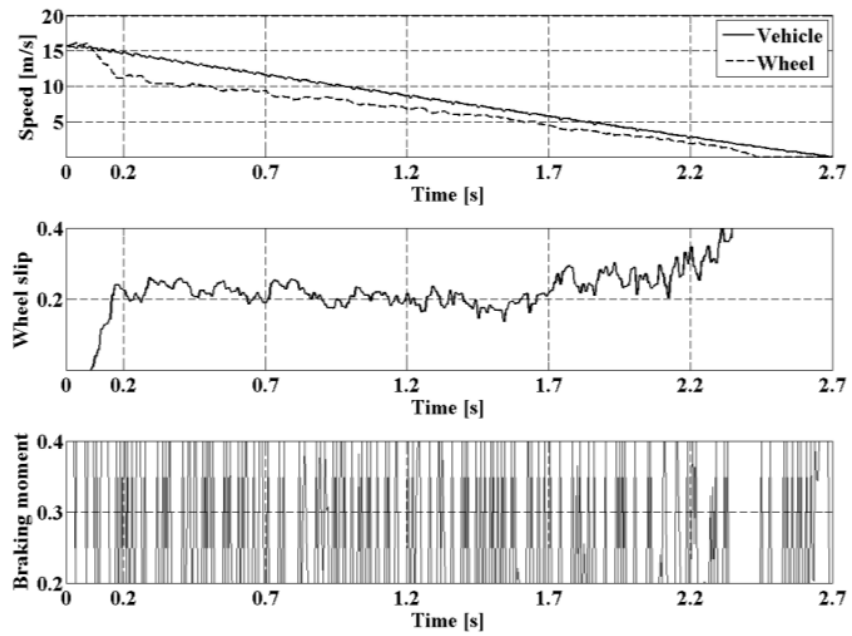

Figure 5

ABS responses with the control law (32) derived from ABS orthogonal model

In both Figures, there are three subplots representing the vehicle and wheel speeds, wheel slip and braking moment, respectively. These three system responses are enough for the valuation of control algorithm. As it can be seen from Figures, the proposed control law suppresses the chattering phenomenon due to the presence of a discrete-time filter in the front of the relay component. In this way, the control law does not lead to excitation of non-modeled high-frequency system dynamics and does not cause deterioration of the mechanical parts of the system.

To make the analysis of the obtained results much easier to explain, the comprehensive index $I$ is introduced as:

$I=2 k_{1} T_{\text {stop }}+k_{2} N+k_{3} E$,

where $k_{1}, k_{2}, k_{3}$ represent the real constant coefficients, $T_{\text {stop }}$ is a stopping time, $N$ is a total number of changes in the control law, and $E$ is an error calculated as $E=\frac{1}{N_{s}} \sum_{i=0}^{N_{s}}\left(\lambda_{k}-\lambda_{k}^{r e f}\right)^{2}$, where $N_{s}$ is a number of samples. It is obvious that all of these values should be as small as possible. The obtained results for the coefficients $k_{1}=0.1984, k_{2}=0.0038, k_{3}=0.0421$ are presented in Table 1 . These values of coefficients provide the normalization of the considered parameters. 
Table 1

Analysis of the obtained experimental results

\begin{tabular}{|l|c|c|c|c|}
\hline Model reference & \multicolumn{1}{|c|}{$T_{\text {stop }}$} & \multicolumn{1}{c|}{$E$} & \multicolumn{1}{c|}{$I$} \\
\hline $\begin{array}{l}\text { Discrete-time } \\
\text { model }\end{array}$ & 2.59 & 147 & 14.56 & 2.20 \\
\cline { 2 - 5 } $\begin{array}{l}\text { Orthogonal } \\
\text { model }\end{array}$ & 2.45 & 113 & 9.19 & 1.79 \\
\hline
\end{tabular}

As it can be seen, the stopping time was slightly shortened when the orthogonal model is used in the controller design. From the tracking point of view, better experimental results are obtained using the orthogonal model, due to a smaller deviation between the current and referent values of wheel slip. In this way, the maximum value for the friction force is constantly ensured leading to the better steering characteristics of the vehicle. A more faithful representation of the plant dynamics comes from the introduced parameter $\delta$, which successfully describes the imperfections in the form of the noise presence, parameter variations, and additional limitation of the control signal to $[0.2,0.4]$ (to avoid the saturation).

\section{Conclusions}

In this paper, a new sliding mode based minimum variance control algorithm designed on the basis of a model obtained by using a new type of orthogonal filters is presented. After giving the necessary background of the used theory, the proposed control law is applied in the anti-lock braking system control. Experimental results have shown that the stopping time, the number in changes in the control law and the tracking error are lesser than in the case where the proposed control is designed on the basis of previously developed ABS model. Having that in mind, it can be concluded that the orthogonal model represents much better system dynamics due to the introduced parameter $\delta$, which characterizes all the imperfections in the system. From the control point of view, both experiments confirm the effectiveness of the proposed robust control method.

\section{Acknowledgement}

This paper was realized as a part of projects TR 35005, III 43007 and III 44006, financed by the Ministry of Education and Science of the Republic of Serbia for the period 2011-2019.

\section{References}

[1] Jingang, G., Xiaoping, J. and Guangyu, L.: Performance evaluation of an anti-lock braking system for electric vehicles with a fuzzy sliding mode controller, Energies, 2014, Vol. 7, No. 10, pp. 6459-6476

[2] Shuwen, Z., Siqi, Z. and Qingming, C.: Vehicle ABS equipped with an EMB system based on the slip ratio control, Transactions of FAMENA, 2019, Vol. 43, No. SI-1, pp. 1-12 
[3] Jun-Cheng, W. and Ren, H.: Hydraulic anti-lock braking control strategy of a vehicle based on a modified optimal sliding mode control method, Proceedings of the Institution of Mechanical Engineers, Part D: Journal of Automobile Engineering, 2018, Vol. 233, No. 12, pp. 3185-3198

[4] Oniz, Y., Kayacan, E. and Kaynak, O.: Simulated and experimental study of antilock braking system using grey sliding mode control, Proceedings of IEEE International Conference on Systems, Man and Cybernetics, Montreal, Que., Canada, 7-10 October 2007, pp. 90-95

[5] Kayacan, E., Oniz, Y. and Kaynak, O.: A grey system modeling approach for sliding-mode control of antilock braking systems, IEEE Transactions on Industrial Electronics, 2009, Vol. 56, No. 8, pp. 3244-3252

[6] Chun, K. and Sunwoo, M.: Wheel slip control with moving sliding surface for traction control system, International Journal of Automotive Technology, 2004, Vol. 5, No. 2, pp. 123-133

[7] Jing, Y., Mao, Y., Dimirovski, G. M., Zheng, Y. and Zhang, S.: Adaptive global sliding mode control strategy for the vehicle antilock braking systems, Proceedings of American Control Conference, St. Louis, MO, USA, 10-12 June 2009, pp. 769-773

[8] Harifi, A., Aghagolzadeh, A., Alizadeh, G. and Sadeghi, M.: Designing a sliding mode controller for slip control of antilock brake systems, Transportation Research Part C: Emerging Technologies, 2008, Vol. 16, No. 6, pp. 731-741

[9] Song, J.: Performance evaluation of a hybrid electric brake system with a sliding mode controller, Mechatronics, 2005, Vol. 15, No. 3, pp. 339-358

[10] Park, E. J., Stoikov, D., Falcao da Luz, L. and Suleman, A.: A performance evaluation of an automotive magnetorheological brake design with a sliding mode controller, Mechatronics, 2006, Vol. 16, No. 7, pp. 405-416

[11] Zheng, S., Tang, H., Han, Z. and Zhang, Y.: Controller design for vehicle stability enhancement, Control Engineering Practice, 2006, Vol. 14, No. 12, pp. 1413-1421

[12] Norhazimi, H., Khairi, A., Yahaya, S., Hazlina, S. and Rozaimi, G.: Second order sliding mode controller for longitudinal wheel slip control, Proceedings of IEEE $8^{\text {th }}$ International Colloquium on Signal Processing and its Applications, Melaka, Malaysia, 23-25 March 2012

[13] Wu, M. and Shih, M.: Using the sliding-mode pwm method in an anti-lock braking system, Asian Journal of Control, 2001, Vol. 3, No. 3, pp. 2553261

[14] Wu, M. and Shih, M.: Simulated and experimental study of hydraulic antilock braking system using sliding-mode PWM control, Mechatronics, 2003, Vol. 13, No. 4, pp. 331-351 
[15] El Hadri, A., Cadiou, J. C. and M'sirdi, N. K.: Adaptive sliding mode control of vehicle traction, Proceedings of $15^{\text {th }}$ Triennial World Congress, Barcelona, Spain, 21-26 July, 2002, Vol. 35, No. 1, pp. 391-396

[16] Nikolić, S., Antić, D., Danković, B., Milojković, M., Jovanović, Z. and Perić, S.: Orthogonal functions applied in antenna positioning, Advances in Electrical and Computer Engineering, 2010, Vol. 10, No. 4, pp. 35-42

[17] Danković, B., Nikolić, S., Milojković, M. and Jovanović, Z.: A class of quasi-orthogonal filters, Journal of Circuits, Systems, and Computers, 2009, Vol. 18, No. 5, pp. 923-931

[18] Milojković, M., Nikolić, S., Danković, B., Antić, D. and Jovanović, Z.: Modeling of dynamical systems based on quasi-orthogonal polynomials, Mathematical and Computer Modeling of Dynamical Systems, 2010, Vol. 16, No. 2, pp. 133-144

[19] Antić, D., Danković, B., Nikolić, S., Milojković, M. and Jovanović, Z.: Approximation based on orthogonal and almost-orthogonal functions, Journal of the Franklin Institute, 2012, Vol. 349, No. 1, pp. 323-336

[20] Milojković, M., Antić, D., Nikolić, S., Jovanović, Z. and Perić, S.: On a new class of quasi-orthogonal filters, International Journal of Electronics, 2013, Vol. 100, No. 10, pp. 1361-1372

[21] Nikolić, S., Antić, D., Perić, S., Danković, N. and Milojković, M.: Design of generalised orthogonal filters: application to the modelling of dynamical systems, International Journal of Electronics, 2016, Vol. 103, No. 2, pp. 269-280

[22] Nikolić, S., Antić, D., Milojković, M., Milovanović, M., Perić, S. and Mitić, D.: Application of neural networks with orthogonal activation functions in control of dynamical systems, International Journal of Electronics, 2016, Vol. 103, No. 4, pp. 667-685

[23] Spasić, M., Mitić, D., Hovd, M. and Antić, D.: Predictive sliding mode control based on Laguerre functions, Journal of Control Engineering and Applied Informatics, 2019, Vol. 21, No. 1, pp. 12-20

[24] Perić, S., Antić, D., Milovanović, M., Mitić, D., Milojković, M. and Nikolić, S.: Quasi-sliding mode control with orthogonal endocrine neural network-based estimator applied in anti-lock braking system, IEEE/ASME Transactions on Mechatronics, 2016, Vol. 21, No. 2, pp. 754-764

[25] Antić, D., Nikolić, S., Milojković, M., Danković, N., Jovanović, Z. and Perić, S.: Sensitivity analysis of imperfect systems using almost orthogonal filters, Acta Polytechnica Hungarica, 2011, Vol. 8, No. 6, pp. 79-94

[26] Milojković, M., Antić, D., Milovanović, M., Nikolić, S., Perić, S. and Almawlawe, M.: Modeling of dynamic systems using orthogonal endocrine 
adaptive neuro-fuzzy inference systems, Journal of Dynamic Systems Measurement and Control, 2015, Vol. 137, No. 9, pp. DS-15-1098

[27] Perić, S., Antić, D., Nikolić, V., Mitić, D., Milojković, M. and Nikolić, S.: A new approach to the sliding mode control design: anti-lock braking system as a case study, Journal of Electrical Engineering, 2014, Vol. 65, No. 1 , pp. $37-43$

[28] Amitava, C., Ranajit, C., Fumitoshi, M. and Takahiro, E.: Augmented stable fuzzy control for flexible robotic arm using LMI approach and neuro-fuzzy state space modeling, IEEE Transactions on Industrial Electronics, 2008, Vol. 55, No. 3, pp. 1256-1270

[29] Haidegger, T., Kovacs, L., Preitl, S., Precup, R. E., Benyo, B. and Benyo, Z.: Controller design solutions for long distance telesurgical applications, International Journal of Artificial Intelligence, 2011, Vol. 6, No. S11, pp. 48-71

[30] Németh, B. and Péter, G.: LPV design for the control of heterogeneous traffic flow with autonomous vehicles, Acta Polytechnica Hungarica, 2019, Vol. 16, No. 7, pp. 233-246

[31] Mitić, D. and Milosavljević, Č.: Sliding mode-based minimum variance and generalized minimum variance controls with $O\left(T^{2}\right)$ and $O\left(T^{3}\right)$ accuracy, Electrical Engineering, 2004, Vol. 86, No. 4, pp. 229-237

[32] Thangavelusamy, D. and Ponnusamy, L.: Elimination of chattering using fuzzy sliding mode controller for drum boiler turbine system, Journal of Control Engineering and Applied Informatics, 2013, Vol. 15, No. 2, pp. 7885

[33] Inteco, The laboratory anti-lock braking system controlled from PC-User's Manual, 2008, available at www.inteco.com.pl

[34] Topalov, A., Oniz, Y., Kayacan, E. and Kaynak, O.: Neuro-fuzzy control of antilock braking system using sliding mode incremental learning algorithm, Neurocomputing, 2011, Vol. 74, No. 11, pp. 1883-1893

[35] Wei, Z. and Xuexun, G.: An ABS control strategy for commercial vehicle, IEEE/ASME Transactions on Mechatronics, 2015, Vol. 20, No. 1, pp. 384392

[36] Lin, C. M. and Hsu, C. F.: Self-learning fuzzy sliding-mode control for antilock braking systems, IEEE Transactions on Control Systems Technology, 2003, Vol. 11, No. 2, pp. 273-278

[37] Mitić, D., Milosavljević, Č. and Veselić, B.: One approach to I/O based design of digital sliding mode control for nonlinear plants, Electronics, 2004, Vol. 8, No. 2, pp. 64-67

[38] Mitić, D.: Digital variable structure systems based on input-output model, University of Niš, Faculty of Electronic Engineering in Niš, 2006 
[39] Mirzaeinejad, H. and Mirzaei, M.: A novel method for non-linear control of wheel slip in anti-lock braking systems, Control Engineering Practice, 2010, Vol. 18, No. 8, pp. 918-926

[40] Martinez-Gardea, M., Mares Guzman, I., Acosta Lua, C., Di Gennaro, S. and Vazquez Alvarez, I.: Design of a nonlinear observer for a laboratory antilock braking system, Journal of Control Engineering and Applied Informatics, 2015, Vol. 17, No. 3, pp. 105-112

[41] Stan, M., Precup, R. E. and Paul, A. S.: Analysis of fuzzy control solutions for anti-lock braking systems, Journal of Control Engineering and Applied Informatics, 2007, Vol. 9, No. 2, pp. 11-22

[42] Mitić, D., Perić, S., Antić, D., Jovanović, Z., Milojković, M. and Nikolić, S.: Digital sliding mode control of anti-lock braking system, Advances in Electrical and Computer Engineering, 2013, Vol. 13, No. 1, pp. 33-40

[43] Precup, R.-E., Preitl, S., Rădac, B. M., Petriu, E. M., Dragoş, C. A. and Tar, J. K.: Experiment-based teaching in advanced control engineering, IEEE Transactions on Education, 2011, Vol. 54, No. 3, pp. 345-355

[44] Utkin, V. I.: Sliding modes in optimization and control, Springer-Verlag, New York, 1992

[45] Zanten, A., Erhardt, R. and Lutz, A. Measurement and simulation of transients in longitudinal and lateral tire forces, SAE Technical Paper 900210,1990 